\title{
EDITORIAL
}

\section{Calcification of bicuspid aortic valves}

\section{M Otto}

Calcific valve disease represents a disease spectrum that ranges from mild irregular valve thickening (or sclerosis) to severe valve obstruction

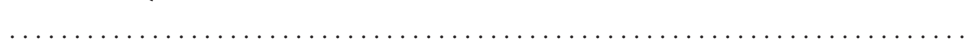

$\mathrm{O}$ ver the past decade, our concept of the pathogenesis of calcific aortic valve disease has undergone a major transformation. We now recognise that calcific valve disease is the end stage of an active disease process and is not caused by "wear and tear" of the valve tissue. Immunohistochemical studies of trileaflet aortic valves with varying degrees of valve stenosis have demonstrated the presence of inflammation, lipid infiltration, and production of proteins that mediate tissue calcification.

These studies have established the presence of T lymphocytes and macrophages both in the early stages of this disease process and in valves with severe stenosis removed at surgery. ${ }^{2}$ The inflammatory cell infiltrate is located in areas of subendothelial lipid infiltration on the aortic side of the valve leaflet with extension into the adjacent fibrosa, the dense collagenous layer of the leaflet that provides tensile strength. The focal presence of neutral lipid can be demonstrated with oil-red-O staining with specific immunohistochemical markers being consistent with infiltration of low density lipoprotein and lipoprotein $\mathrm{Lp}(\mathrm{a})$. In addition, there is evidence of lipid oxidation, as is seen in atherosclerotic lesions. ${ }^{34}$ The interrelations between lipid infiltration, oxidation, and inflammation appear to be similar to the process of atherosclerosis.

In addition, several proteins associated with tissue calcification have been localised in sclerotic and stenotic trileaflet aortic valves. In vitro hybridisation has shown that osteopontin is produced by a subset of lesion macrophages, while bone morphogenetic proteins 2 and 4 are expressed by myofibroblasts, in areas adjacent to lymphocyte infiltration. ${ }^{56}$ Other evidence of an active disease process in calcific aortic valve disease includes up-regulation of adhesion molecules, expression of matrix metalloproteinase, and production of the large extracellular matrix glycoprotein, tenascin-C. ${ }^{7-10}$

\section{AORTIC STENOSIS}

This disease process eventually leads to increased Correspondence to: Professor Catherine $M$ Otto, Division of Cardiology, Box 356422, University of Washington, Seattle, WA 98195-6422, USA; cmotto@

u.washington.edu nosis. In patients with an anatomically normal trileaflet aortic valve, this process occurs in only a subset of patients with symptoms typically occurring at age $70-80$ years. Clinical factors associated with atherosclerosis are also associated with calcific aortic valve disease, including age, sex, hypertension, raised serum cholesterol concentration, smoking, and diabetes. ${ }^{11}{ }^{12}$ However, the specific factors that initiate this disease process have not been well defined. By analogy with atherosclerosis, our underlying assumption is that endothelial disruption occurs, possibly caused by low shear stress on the aortic side of the valve. Low shear stress might explain why the calcific process often is seen first and most severely on the non-coronary cusp.

Mechanical or tensile stress on the valve leaflets also has been proposed as an important factor in initiation of the disease process. The major evidence for this hypothesis is that calcific disease occurs at an earlier age in patients with a bicuspid valve, typically with symptoms occurring at 50-60 years of age. ${ }^{13}$ Also, in contrast to the small number of patients with a trileaflet valve that develop severe stenosis, nearly all patients with a bicuspid valve will develop significant outflow tract obstruction. Because the stress-strain relations of a bicuspid valve are abnormal, this higher tensile stress may contribute to earlier initiation and more rapid progression of disease. However, the disease process at the tissue level in bicuspid aortic valves has not been specifically examined, until now.

In this issue of Heart, the study by Wallby and colleagues $^{14}$ is the first to compare the disease process at the tissue level in bicuspid and in trileaflet valves in patients with severe stenosis undergoing aortic valve replacement. They observed that $\mathrm{T}$ lymphocyte infiltration was present in both bicuspid and trileaflet aortic valves, with a similar pattern and extent of $\mathrm{T}$ lymphocytes in the stenotic valve leaflets. Their observations confirm the hypothesis that the disease process is the same in trileaflet and bicuspid valves, again suggesting the differences in clinical presentation are related to the mechanical characteristics of the valve. Although this study examined only valves removed at surgery, extrapolating from previous studies on trileaflet valves, severe calcific valve disease represents the extreme end of a disease spectrum that ranges from mild irregular valve thickening (or sclerosis) to severe valve obstruction.

\section{CLINICAL IMPLICATIONS}

These findings have important clinical implications. A bicuspid aortic valve is present in $1-2 \%$ of the population and most of these patients will eventually develop stenosis, requiring valve surgery. A minority of patients develop aortic regurgitation as a young adult but the majority have progressive valve stenosis, with only about $1 \%$ maintaining normal valve function over their 
lifetime. ${ }^{13}$ Many of these patients are diagnosed early in life, based on the physical finding of an ejection click or systolic murmur, with echocardiography allowing reliable identification of the number of valve leaflets. There may be a genetic predisposition to a bicuspid valve, possibly allowing family screening. ${ }^{15}$ If an effective treatment to prevent inflammation and lipid deposition in the valve leaflets were available, patients with a bicuspid valve could begin treatment at the time of diagnosis, delaying or avoiding the need for valve replacement surgery. At this time, such an intervention is not available, although our understanding of the disease process at the tissue level and the known clinical associations suggest that treatment directed at the disease process in the leaflets may be possible in the future. ${ }^{16}$

In the meanwhile, since the disease process in bicuspid and trileaflet valves appear similar and because most patients with a bicuspid valve eventually need surgery, aggressive evaluation and treatment of conventional coronary risk factors is indicated in all patients with a bicuspid aortic valve. Although the effect of this treatment on the aortic valve itself will not be known until randomised clinical trials are performed, at the very least we will be decreasing the likelihood that concurrent coronary artery bypass grafting will be needed at the time of valve replacement.

\section{REFERENCES}

1 Otto CM, Kuusisto J, Reichenbach DD, et al. Characterization of the early lesion of 'degenerative' valvular aortic stenosis: histologic and immunohistochemical studies. Circulation 1994;90:844-53.

2 Olsson M. Dalsgaard CJ, Haegerstrand A, et al. Accumulation of T lymphocytes and expression of interleukin-2 receptors in nonrheumatic stenotic aortic valves. J Am Coll Cardiol 1994;23: 1 162-70.
3 Olsson M, Thyberg J, Nilsson J. Presence of oxidized low density lipoprotein in nonrheumatic stenotic aortic valves. Arterioscler Thromb Vasc Biol 1999;19:1218-22.

4 O'Brien KD, Reichenbach DD, Marcovina SM, et al. Apolipoproteins B, (a) and $E$ accumulate in the morphologically early lesion of "degenerative" valvular aortic stenosis. Arterioscler Thromb 1996; 16:523-32.

5 O'Brien KD, Kuusisto J, Reichenbach DD, et al. Osteopontin is expressed in human aortic valvular lesions. Circulation 1995;92:2163-8.

6 Mohler ER, III, Gannon F, Reynolds C, et al. Bone formation and inflammation in cardiac valves. Circulation $2001 ; 103: 1522-8$.

7 Ghaisas NK, Foley JB, O'Briain DS, et al. Adhesion molecules in nonrheumatic aortic valve disease: endothelial expression, serum levels and effects of valve replacement. J Am Coll Cardiol 2000;36:2257-62.

8 Edep ME, Shirani J, Wolf $\mathrm{P}$, et al. Matrix metalloproteinase expression in nonrheumatic aortic stenosis. Cardiovasc Pathol 2000;9:281-6.

9 Muller AM, Cronen C, Kupferwasser LI, et al. Expression of endothelial cell adhesion molecules on heart valves: up- regulation in degeneration as well as acute endocarditis. J Pathol 2000;191:54-60.

10 Jian B, Jones PL, Li Q, et al. Matrix metalloproteinase-2 is associated with tenascin-C in calcific aortic stenosis. Am J Pathol 2001;159:321-7.

11 Stewart BF, Siscovick D, Lind BK, et al. Clinical factors associated with calcific aortic valve disease. J Am Coll Cardiol 1997;29:630-4.

12 Boon A, Cheriex E, Lodder J, et al. Cardiac valve calcification: characteristics of patients with calcification of the mitral annulus or aortic valve. Heart 1997:78:472-4.

13 Beppu S, Suzuki S, Matsuda H, et al. Rapidity of progression of aortic stenosis in patients with congenital bicuspid aortic valves. Am J Cardiol 1993;71:322-7.

14 Wallby L, Janerot-Sjöberg B, Steffensen T, et al. T lymphocyte infiltration in non-rheumatic aortic stenosis: a comparative descriptive study between tricuspid and bicuspid aortic valves. Heart 2002;88:348-51.

15 Huntington K, Hunter AG, Chan KL. A prospective study to assess the frequency of familial clustering of congenital bicuspid aortic valve. J Am Coll Cardiol 1997;30:1809-12.

16 Chan KL, Ghani M, Woodend K, et al. Case-controlled study to assess risk factors for aortic stenosis in congenitally bicuspid aortic valve. Am J Cardiol 2001;88:690-3.

\section{IMAGES IN CARDIOLOGY}

\section{Anomalous origin of left anterior descending and circumflex coronary artery from two separate ostia in the right aortic sinus}

A

63 year old woman presented with atypical chest pain. A 12 lead ECG showed complete left bundle brunch block; the echocardiogram was not diagnostic. At coronary angiography the left coronary ostium was not observed. Aortography showed the left anterior descending (LAD) artery and the circumflex $(\mathrm{CX})$ artery each originating from a separate ostium in the right aortic sinus. The right coronary artery (RCA) had a normal independent origin. The three vessels were then selectively engaged (the LAD with an internal mammary catheter, the $\mathrm{CX}$ with a multipurpose catheter, the RCA with a right Judkins catheter), resulting in the patient being free from atherosclerotic disease. Subsequent stress myocardial perfusion imaging was negative for inducible ischaemia. The patient had an uneventful two year follow up.

The independent origin of both the LAD and CX from two separate ostia in the right aortic sinus is an exceedingly rare anomaly. The hazard of this coronary anomaly depends on the LAD course. An LAD course between the aorta and the pulmonary artery is potentially responsible for myocardial ischaemia and sudden death, while an LAD course anterior to the right ventricle (like the present case), or behind the aorta, is at low risk of adverse events. In this regard, the scintigraphic findings obtained in our patient confirmed the benign prognosis associated with an anterior free wall course of the LAD.
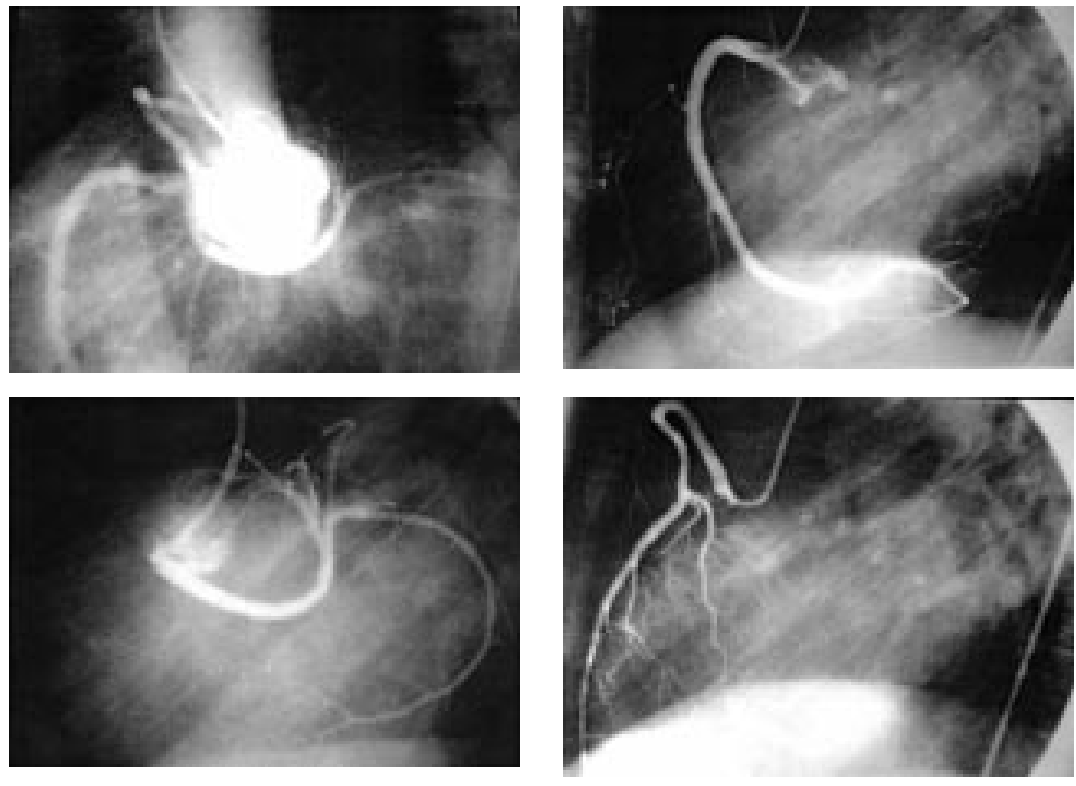

L La Vecchia

L Favero

A Fontanelli

luigilavecchia@libero.it 